PROCEEDINGS OF THE

AMERICAN MATHEMATICAL SOCIETY

Volume 137, Number 6, June 2009, Pages 1891-1898

S 0002-9939(09)09825-6

Article electronically published on January 26, 2009

\title{
THE GROUP OF ORDER PRESERVING AUTOMORPHISMS OF THE RING OF DIFFERENTIAL OPERATORS ON A LAURENT POLYNOMIAL ALGEBRA IN PRIME CHARACTERISTIC
}

\author{
V. V. BAVULA
}

(Communicated by Martin Lorenz)

\begin{abstract}
Let $K$ be a field of characteristic $p>0$. It is proved that the group Aut ord $\left(\mathcal{D}\left(L_{n}\right)\right)$ of order preserving automorphisms of the ring $\mathcal{D}\left(L_{n}\right)$ of differential operators on a Laurent polynomial algebra $L_{n}:=K\left[x_{1}^{ \pm 1}, \ldots, x_{n}^{ \pm 1}\right]$ is isomorphic to a skew direct product of groups $\mathbb{Z}_{p}^{n} \rtimes \operatorname{Aut}_{K}\left(L_{n}\right)$, where $\mathbb{Z}_{p}$ is the ring of $p$-adic integers. Moreover, the group Aut $\operatorname{Ard}\left(\mathcal{D}\left(L_{n}\right)\right)$ is found explicitly. Similarly, Aut ord $\left(\mathcal{D}\left(P_{n}\right)\right) \simeq \operatorname{Aut}_{K}\left(P_{n}\right)$, where $P_{n}:=K\left[x_{1}, \ldots, x_{n}\right]$ is a polynomial algebra.
\end{abstract}

\section{INTRODUCTION}

Throughout, ring means an associative ring with $1 ; p$ is a prime number; $\mathbb{F}_{p}:=$ $\mathbb{Z} / p \mathbb{Z}$ is the field that contains $p$ elements; $\mathbb{Z}_{p}$ is the ring of $p$-adic integers; $K$ is an arbitrary field of characteristic $p>0$ (if it is not stated otherwise); $P_{n}:=$ $K\left[x_{1}, \ldots, x_{n}\right]$ is a polynomial algebra; $\mathcal{D}\left(P_{n}\right)=\bigoplus_{\alpha \in \mathbb{N}^{n}} P_{n} \partial^{[\alpha]}$ is the ring of differential operators on $P_{n}$ where $\partial^{[\alpha]}:=\prod_{i=1}^{n} \frac{\partial_{i}^{\alpha_{i}}}{\alpha_{i} !} ; L_{n}:=K\left[x_{1}^{ \pm 1}, \ldots, x_{n}^{ \pm 1}\right]$ is a Laurent polynomial algebra and $\mathcal{D}\left(L_{n}\right)=\bigoplus_{\alpha \in \mathbb{N} n} L_{n} \partial^{[\alpha]}$ is the ring of differential operators on the algebra $L_{n} ;\left\{\mathcal{D}\left(L_{n}\right)_{i}:=\bigoplus_{|\alpha|<i} L_{n} \partial^{[\alpha]}\right\}_{i \geq 0}$ is the order filtration on $\mathcal{D}\left(L_{n}\right)$; and

$$
\operatorname{Aut}_{\text {ord }}\left(\mathcal{D}\left(L_{n}\right)\right):=\left\{\sigma \in \operatorname{Aut}_{K}\left(\mathcal{D}\left(L_{n}\right)\right) \mid \sigma\left(\mathcal{D}\left(L_{n}\right)_{i}\right)=\mathcal{D}\left(L_{n}\right)_{i}, i \geq 0\right\}
$$

is the group of order preserving automorphisms of the algebra $\mathcal{D}\left(L_{n}\right)$. Similarly the order filtration on $\mathcal{D}\left(P_{n}\right)$ and the group $\mathrm{Aut}_{\text {ord }}\left(\mathcal{D}\left(P_{n}\right)\right)$ are defined.

In arbitrary characteristic, it is a difficult problem to find generators for the groups $\operatorname{Aut}_{K}\left(P_{n}\right)$ and $\operatorname{Aut}_{K}\left(\mathcal{D}\left(P_{n}\right)\right)$. The results are known for $\operatorname{Aut}_{K}\left(P_{1}\right)$ (easy), $\operatorname{Aut}_{K}\left(P_{2}\right)$ (Jung [8] and van der Kulk [10]) and $\operatorname{Aut}_{K}\left(\mathcal{D}\left(P_{1}\right)\right)$ when $\operatorname{char}(K)=0$ (Dixmier [7). Little is known about the groups of automorphisms in the remaining cases.

In characteristic zero, there is a strong connection between the $\operatorname{groups}^{\operatorname{Aut}_{K}}\left(P_{n}\right)$ and $\operatorname{Aut}_{K}\left(\mathcal{D}\left(P_{n}\right)\right)$ as, for example, the (essential) equivalence of the Jacobian Conjecture for $P_{n}$ and the Dixmier Problem/Conjecture for $\mathcal{D}\left(P_{n}\right)$ shows (see [1, 9,

Received by the editors June 4, 2008.

2000 Mathematics Subject Classification. Primary 16W20, 13N10, 16S32.

Key words and phrases. Group of automorphisms, ring of differential operators, the order filtration. 
6]; see also [4). Moreover, conjectures such as the two mentioned conjectures make sense only for the algebras $P_{m} \otimes \mathcal{D}\left(P_{n}\right)$ as was proved in [2] (the two conjectures can be reformulated in terms of locally nilpotent derivations that satisfy certain conditions, and the algebras $P_{m} \otimes \mathcal{D}\left(P_{n}\right)$ are the only associative algebras that have such derivations). This general conjecture is true iff either the JC or the DC is true; see [2].

In prime characteristic, relations between the two groups $\operatorname{Aut}_{K}\left(P_{n}\right)$ and $\operatorname{Aut}_{K}\left(\mathcal{D}\left(P_{n}\right)\right)$ are even tighter, as the following result shows.

Theorem 1.1 ( $\left[3\right.$, Rigidity of the group $\left.\operatorname{Aut}_{K}\left(\mathcal{D}\left(P_{n}\right)\right)\right)$. Let $K$ be a field of characteristic $p>0$ and $\sigma, \tau \in \operatorname{Aut}_{K}\left(\mathcal{D}\left(P_{n}\right)\right)$. Then $\sigma=\tau$ iff $\sigma\left(x_{1}\right)=\tau\left(x_{1}\right), \ldots, \sigma\left(x_{n}\right)=$ $\tau\left(x_{n}\right)$.

Remark. Theorem 1.1 does not hold in characteristic zero, and, in general, in prime characteristic it does not hold for localizations of the polynomial algebra $P_{n}$ (Theorem 1.3). Note that the $K$-algebra $\mathcal{D}\left(P_{n}\right)$ is not finitely generated.

As a direct consequence of Theorem 1.1 there is the corollary (see Section 2 for details).

Corollary 1.2. Aut $_{\text {ord }}\left(\mathcal{D}\left(P_{n}\right)\right) \simeq \operatorname{Aut}_{K}\left(P_{n}\right)$.

The situation is completely different for the Laurent polynomial algebra $L_{n}$.

Theorem 1.3. Aut ord $\left(\mathcal{D}\left(L_{n}\right)\right) \simeq \mathbb{Z}_{p}^{n} \rtimes \operatorname{Aut}_{K}\left(L_{n}\right)$. Moreover, each automorphism $\sigma \in \operatorname{Aut}_{\text {ord }}\left(\mathcal{D}\left(L_{n}\right)\right)$ is a unique product of two automorphisms $\sigma=\sigma_{s} \tau$ where $\tau \in \operatorname{Aut}_{K}\left(L_{n}\right), s=\left(s_{i}\right) \in \mathbb{Z}_{p}^{n}, \sigma_{s}\left(x_{i}\right)=x_{i}$ for all $i$, and

$$
\sigma_{s}\left(\partial^{[\alpha]}\right)=\prod_{i=1}^{n} \frac{\left(\partial_{i}+s_{i} x_{i}^{-1}\right)^{\alpha_{i}}}{\alpha_{i} !}, \alpha=\left(\alpha_{i}\right) \in \mathbb{N}^{n} .
$$

The meaning of the RHS of (1) is explained in Section 2, Theorem 1.3 gives all the elements of the group $\operatorname{Aut}_{\text {ord }}\left(\mathcal{D}\left(L_{n}\right)\right)$ explicitly since the group $\operatorname{Aut}_{K}\left(L_{n}\right)$ is known: it is isomorphic to the semidirect product $\mathrm{GL}_{n}(\mathbb{Z}) \rtimes K^{* n}$.

The main idea of the proof of Theorem 1.3 is to show that the group Aut $_{\text {ord }}\left(\mathcal{D}\left(L_{n}\right)\right)$ is equal to the group

$$
\operatorname{St}\left(L_{n}\right):=\left\{\sigma \in \operatorname{Aut}_{K}\left(\mathcal{D}\left(L_{n}\right)\right) \mid \sigma\left(L_{n}\right)=L_{n}\right\},
$$

which is a semidirect product $\operatorname{St}\left(L_{n}\right)=\operatorname{st}\left(L_{n}\right) \rtimes \operatorname{Aut}_{K}\left(L_{n}\right)$, where

$$
\operatorname{st}\left(L_{n}\right):=\left\{\sigma \in \operatorname{St}\left(L_{n}\right)|\sigma|_{L_{n}}=\mathrm{id}\right\}
$$

is the normal subgroup of $\operatorname{St}\left(L_{n}\right)$. The group $\operatorname{st}\left(L_{n}\right)$ consists of all the automorphisms $\sigma_{s}, s \in \mathbb{Z}_{p}^{n}$ (see (11)), and the map $\mathbb{Z}_{p}^{n} \rightarrow \operatorname{st}\left(L_{n}\right), s \mapsto \sigma_{s}$, is a group isomorphism $\left(\sigma_{s+t}=\sigma_{s} \sigma_{t}\right)$; see Theorem 2.4.

\section{The GRoup $\operatorname{Aut}_{\text {ord }}\left(\mathcal{D}\left(L_{n}\right)\right)$}

Let $R$ be a commutative $K$-algebra and $\mathcal{D}(R)=\bigcup_{i>0} \mathcal{D}(R)_{i}$ be the ring of $K$-linear differential operators on the algebra $R$, where $\left\{\mathcal{D}(R)_{i}\right\}_{i \geq 0}$ is the order filtration on $\mathcal{D}(R)$. In more detail, $\mathcal{D}(R) \subseteq \operatorname{End}_{K}(R), \mathcal{D}(R)_{0}:=\operatorname{End}_{R}(R) \simeq R$, and

$$
\mathcal{D}(R)_{i}:=\left\{f \in \operatorname{End}_{K}(R) \mid r f-f r \in \mathcal{D}(R)_{i-1} \text { for all } r \in R\right\}, \quad i \geq 1 .
$$


Let $\operatorname{Aut}_{\text {ord }}(\mathcal{D}(R))$ be the subgroup of $\operatorname{Aut}_{K}(\mathcal{D}(R))$ of order preserving $K$-automorphisms, i.e.

$$
\operatorname{Aut}_{\text {ord }}(\mathcal{D}(R)):=\left\{\sigma \in \operatorname{Aut}_{K}(\mathcal{D}(R)) \mid \sigma\left(\mathcal{D}(R)_{i}\right)=\mathcal{D}(R)_{i}, i \geq 0\right\} .
$$

Each automorphism $\sigma \in \operatorname{Aut}_{K}(R)$ can be naturally extended (by change of variables) to a $K$-automorphism, say $\sigma$, of the ring $\mathcal{D}(R)$ of differential operators on the algebra $R$ by the rule

$$
\sigma(a):=\sigma a \sigma^{-1}, \quad a \in \mathcal{D}(R)
$$

Then the group $\operatorname{Aut}_{K}(R)$ can be seen as a subgroup of $\operatorname{Aut}_{K}(\mathcal{D}(R))$ via (2). Then it follows from a definition of the ring $\mathcal{D}(R)$ and the equality $\left[r, \sigma a \sigma^{-1}\right]=$ $\sigma\left[\sigma^{-1}(r), a\right] \sigma^{-1}$, where $r \in R, a \in \mathcal{D}(R)$, that

$$
\operatorname{Aut}_{K}(R) \subseteq \operatorname{Aut}_{\text {ord }}(\mathcal{D}(R))
$$

The stabilizer of the algebra $R$,

$$
\operatorname{St}(R):=\left\{\sigma \in \operatorname{Aut}_{K}(\mathcal{D}(R)) \mid \sigma(R)=R\right\},
$$

is a subgroup of $\operatorname{Aut}_{K}(\mathcal{D}(R))$. It contains the normal subgroup

$$
\operatorname{st}(R):=\left\{\sigma \in \operatorname{St}(R)|\sigma|_{R}=\mathrm{id}\right\},
$$

which is the kernel of the restriction epimorphism

$$
\operatorname{St}(R) \rightarrow \operatorname{Aut}_{K}(R),\left.\quad \sigma \mapsto \sigma\right|_{R}
$$

By (3), the stabilizer of $R$,

$$
\operatorname{St}(R)=\operatorname{st}(R) \rtimes \operatorname{Aut}_{K}(R)
$$

is the semi-direct product of its subgroups. It is obvious that

$$
\text { Aut }_{\text {ord }}(\mathcal{D}(R)) \subseteq \operatorname{St}(R) .
$$

The ring $R$ is a left $\mathcal{D}(R)$-module. The action of an element $\delta \in \mathcal{D}(R)$ on an element $r \in R$ is denoted either by $\delta(a)$ or $\delta * r$ (in order to avoid multiple brackets).

Proof of Corollary 1.2, By Theorem 1.1, $\operatorname{st}\left(P_{n}\right)=\{\operatorname{id}\}$. Now the result follows from (3), (4) and (5):

$$
\operatorname{Aut}_{K}\left(P_{n}\right) \subseteq \operatorname{Aut}_{\text {ord }}\left(\mathcal{D}\left(P_{n}\right)\right) \subseteq \operatorname{St}\left(P_{n}\right)=\operatorname{st}\left(P_{n}\right) \rtimes \operatorname{Aut}_{K}\left(P_{n}\right)=\operatorname{Aut}_{K}\left(P_{n}\right) .
$$

The rings of differential operators $\mathcal{D}\left(P_{n}\right)$ and $\mathcal{D}\left(L_{n}\right)$. The ring $\mathcal{D}\left(P_{n}\right)$ of differential operators on a polynomial algebra $P_{n}:=K\left[x_{1}, \ldots, x_{n}\right]$ is a $K$-algebra generated by the elements $x_{1}, \ldots, x_{n}$ and commuting higher derivations $\partial_{i}^{[k]}:=\frac{\partial_{i}^{k}}{k !}$, $i=1, \ldots, n, k \geq 1$, that satisfy the following defining relations:

(6) $\left[x_{i}, x_{j}\right]=0, \quad\left[\partial_{i}^{[k]}, \partial_{j}^{[l]}\right]=0, \quad \partial_{i}^{[k]} \partial_{i}^{[l]}=\left(\begin{array}{c}k+l \\ k\end{array}\right) \partial_{i}^{[k+l]}, \quad\left[\partial_{i}^{[k]}, x_{j}\right]=\delta_{i j} \partial_{i}^{[k-1]}$,

for all $i, j=1, \ldots, n, k, l \geq 1$, where $\delta_{i j}$ is the Kronecker delta, $\partial_{i}^{[0]}:=1, \partial_{i}^{[-1]}:=0$, and $\partial_{i}^{[1]}=\partial_{i}=\frac{\partial}{\partial x_{i}} \in \operatorname{Der}_{K}\left(P_{n}\right), i=1, \ldots, n$. The action of the higher derivation $\partial_{i}^{[k]}$ on the polynomial algebra

$$
P_{n}=K \otimes_{\mathbb{Z}} \mathbb{Z}\left[x_{1}, \ldots, x_{n}\right] \simeq K \otimes_{\mathbb{Z}} \mathbb{Z}_{p}\left[x_{1}, \ldots, x_{n}\right]
$$

should be understood as the action of the element $1 \otimes_{\mathbb{Z}} \frac{\partial_{i}^{k}}{k !}$. 
The algebra $\mathcal{D}\left(P_{n}\right)$ is a simple algebra. Note that the algebra $\mathcal{D}\left(P_{n}\right)$ is not finitely generated, is not (left or right) Noetherian, and does not satisfy finitely many defining relations. This is in contrast to the characteristic zero case. We have

$$
\mathcal{D}\left(P_{n}\right)=\bigoplus_{\alpha, \beta \in \mathbb{N}^{n}} K x^{\alpha} \partial^{[\beta]} \subset \mathcal{D}\left(L_{n}\right)=\bigoplus_{\alpha \in \mathbb{Z}^{n}, \beta \in \mathbb{N}^{n}} K x^{\alpha} \partial^{[\beta]} .
$$

For each $i=1, \ldots, n$ and $j \in \mathbb{N}$ written $p$-adically as $j=\sum_{k} j_{k} p^{k}, 0 \leq j_{k}<p$,

$$
\partial_{i}^{[j]}=\prod_{k} \partial_{i}^{\left[j_{k} p^{k}\right]}=\prod_{k} \frac{\partial_{i}^{\left[p^{k}\right] j_{k}}}{j_{k} !}, \quad \partial_{i}^{\left[j_{k} p^{k}\right]}=\frac{\partial_{i}^{\left[p^{k}\right] j_{k}}}{j_{k} !},
$$

where $\partial_{i}^{\left[p^{k}\right] j_{k}}:=\left(\partial_{i}^{\left[p^{k}\right]}\right)^{j_{k}}$. For $\alpha \in \mathbb{N}^{n}$ and $\beta \in \mathbb{Z}^{n}$,

$$
\partial^{[\alpha]} * x^{\beta}=\left(\begin{array}{c}
\beta \\
\alpha
\end{array}\right) x^{\beta-\alpha}, \quad\left(\begin{array}{c}
\beta \\
\alpha
\end{array}\right):=\prod_{i}\left(\begin{array}{c}
\beta_{i} \\
\alpha_{i}
\end{array}\right) .
$$

For $\alpha, \beta \in \mathbb{N}^{n}$,

$$
\partial^{[\alpha]} \partial^{[\beta]}=\left(\begin{array}{c}
\alpha+\beta \\
\beta
\end{array}\right) \partial^{[\alpha+\beta]} .
$$

The binomial differential operators. For each natural number $i$, the binomial polynomial

$$
\left(\begin{array}{l}
t \\
i
\end{array}\right):=\frac{t(t-1) \cdots(t-i+1)}{i !},\left(\begin{array}{l}
t \\
0
\end{array}\right):=1
$$

can be seen as a function from $\mathbb{Z}_{p}$ to $\mathbb{Z}_{p}$. For each $p$-adic integer $s \in \mathbb{Z}_{p}$ and a natural number $i \in \mathbb{N}$, we have the differential operator on the $\mathbb{Z}_{p}$-algebra $\mathbb{Z}_{p}\left[x^{ \pm 1}\right]:=$ $\mathbb{Z}_{p}\left[x, x^{-1}\right]$ of Laurent polynomials with coefficients from $\mathbb{Z}_{p}$ :

$$
\begin{aligned}
\frac{\left(\partial+s x^{-1}\right)^{i}}{i !} & =x^{-i} \frac{(x \partial+s)(x \partial+s-1) \cdots(x \partial+s-i+1)}{i !} \\
& =x^{-i}\left(\begin{array}{c}
x \partial+s \\
i
\end{array}\right) \in \mathcal{D}\left(\mathbb{Z}_{p}\left[x^{ \pm 1}\right]\right),
\end{aligned}
$$

where $\partial:=\frac{d}{d x} \in \operatorname{Der}_{\mathbb{Z}_{p}}\left(\mathbb{Z}_{p}\left[x^{ \pm 1}\right]\right)$. Let $\mathcal{L}_{n}:=\mathbb{Z}_{p}\left[x_{1}^{ \pm 1}, \ldots, x_{n}^{ \pm 1}\right]$ be the Laurent polynomial ring in $n$ variables with coefficients from $\mathbb{Z}_{p}$ and let $\mathcal{D}\left(\mathcal{L}_{n}\right)$ be the ring of $\mathbb{Z}_{p}$-linear differential operators on the ring $\mathcal{L}_{n}$. Then, for any elements $\alpha=\left(\alpha_{i}\right) \in \mathbb{N}^{n}$ and $s=\left(s_{i}\right) \in \mathbb{Z}_{p}^{n}$, there is the following differential operator on the algebra $\mathcal{L}_{n}$ :

$$
b_{s}^{[\alpha]}:=\prod_{i=1}^{n} x_{i}^{-\alpha_{i}}\left(\begin{array}{c}
x_{i} \partial_{i}+s_{i} \\
\alpha_{i}
\end{array}\right)=\prod_{i=1}^{n} \frac{\left(\partial_{i}+s_{i} x_{i}^{-1}\right)^{\alpha_{i}}}{\alpha_{i} !} \in \mathcal{D}\left(\mathcal{L}_{n}\right) .
$$

The inclusions of abelian monoids $\mathbb{N}^{n} \subset \mathbb{Z}^{n} \subset \mathbb{Z}_{p}^{n}$ yield the inclusions of their monoid rings

$$
\mathcal{N}_{n}:=\bigoplus_{\alpha \in \mathbb{N}^{n}} \mathbb{Z}_{p} x^{\alpha} \subset \mathcal{L}_{n} \subset \mathcal{M}_{n}:=\bigoplus_{\alpha \in \mathbb{Z}_{p}^{n}} \mathbb{Z}_{p} x^{\alpha},
$$

and the inclusions of rings

$$
\mathcal{A}_{n}:=\bigoplus_{\beta \in \mathbb{N}^{n}} \mathcal{N}_{n} \partial^{[\beta]} \subset \mathcal{B}_{n}:=\bigoplus_{\beta \in \mathbb{N}^{n}} \mathcal{L}_{n} \partial^{[\beta]} \subset \mathcal{C}_{n}:=\bigoplus_{\beta \in \mathbb{N}^{n}} \mathcal{M}_{n} \partial^{[\beta]} \subset \operatorname{End}_{\mathbb{Z}_{p}}\left(\mathcal{M}_{n}\right),
$$


where $\partial^{[\beta]}=\prod_{i=1}^{n} \frac{\partial_{i}^{\beta_{i}}}{\beta_{i} !}$. For any elements $\alpha \in \mathbb{Z}_{p}^{n}$ and $\beta \in \mathbb{N}^{n}$

$$
\partial^{[\beta]} * x^{\alpha}=\left(\begin{array}{c}
\alpha \\
\beta
\end{array}\right) x^{\alpha-\beta}, \text { where }\left(\begin{array}{l}
\alpha \\
\beta
\end{array}\right):=\prod_{i=1}^{n}\left(\begin{array}{c}
\alpha_{i} \\
\beta_{i}
\end{array}\right) .
$$

The prime number $p$ belongs to the centre of each of the $\operatorname{rings} \mathcal{A}_{n}, \mathcal{B}_{n}$ and $\mathcal{C}_{n}$. Taking factor rings modulo the ideals generated by the element $p$ in each of the rings, we obtain the inclusions of $\mathbb{F}_{p}$-algebras:

$$
\bigoplus_{\alpha, \beta \in \mathbb{N}^{n}} \mathbb{F}_{p} x^{\alpha} \partial^{[\beta]} \subset \bigoplus_{\alpha \in \mathbb{Z}^{n}, \beta \in \mathbb{N}^{n}} \mathbb{F}_{p} x^{\alpha} \partial^{[\beta]} \subset \bigoplus_{\alpha \in \mathbb{Z}_{p}^{n}, \beta \in \mathbb{N}^{n}} \mathbb{F}_{p} x^{\alpha} \partial^{[\beta]} \subset \operatorname{End}_{\mathbb{F}_{p}}\left(\bigoplus_{\alpha \in \mathbb{Z}^{n}} \mathbb{F}_{p} x^{\alpha}\right) .
$$

Then, applying $K \otimes_{\mathbb{Z}}-$, we obtain the inclusions of rings

$$
\mathcal{D}\left(P_{n}\right) \subset \mathcal{D}\left(L_{n}\right) \subset \mathcal{D}_{n}:=\bigoplus_{\alpha \in \mathbb{Z}_{p}^{n}, \beta \in \mathbb{N}^{n}} K x^{\alpha} \partial^{[\beta]} .
$$

For each element $s \in \mathbb{Z}_{p}^{n}$, the inner automorphism $\omega_{x^{-s}}: a \mapsto x^{-s} a x^{s}$ of the ring $\mathcal{C}_{n}$ acts trivially on the ring $\mathcal{M}_{n}$, and, for each element $\beta \in \mathbb{N}^{n}$,

$$
\omega_{x^{-s}}\left(\partial^{[\beta]}\right)=b_{s}^{[\beta]} \text {. }
$$

Indeed,

$$
\begin{aligned}
\omega_{x^{-s}}\left(\partial^{[\beta]}\right) & =\prod_{i=1}^{n} x_{i}^{-s_{i}} \partial_{i}^{\left[\beta_{i}\right]} x_{i}^{s_{i}}=\prod_{i=1}^{n} x_{i}^{-s_{i}} x_{i}^{-\beta_{i}} \frac{x_{i}^{\beta_{i}} \partial_{i}^{\beta_{i}}}{\beta_{i} !} x_{i}^{s_{i}}=\prod_{i=1}^{n} x_{i}^{-\beta_{i}} x_{i}^{-s_{i}}\left(\begin{array}{c}
x_{i} \partial_{i} \\
\beta_{i}
\end{array}\right) x_{i}^{s_{i}} \\
& =\prod_{i=1}^{n} x_{i}^{-\beta_{i}}\left(\begin{array}{c}
x_{i} \partial_{i}+s_{i} \\
\beta_{i}
\end{array}\right) x_{i}^{-s_{i}} x_{i}^{s_{i}}=b_{s}^{[\beta]} .
\end{aligned}
$$

The inner automorphism $\omega_{x^{-s}}: b \mapsto x^{-s} b x^{s}$ of the ring $\mathcal{D}_{n}$ is the restriction modulo $p$ of the inner automorphism $\omega_{x^{-s}}: a \mapsto x^{-s} a x^{s}$ of the $\operatorname{ring} \mathcal{C}_{n}$. It is obvious that

$$
\omega_{x^{-s}}\left(\mathcal{D}\left(L_{n}\right)\right)=\mathcal{D}\left(L_{n}\right) \text {. }
$$

Let $\sigma_{s}$ be the restriction of the inner automorphism $\omega_{x^{-s}}$ of the ring $\mathcal{D}_{n}$ to the subring $\mathcal{D}\left(L_{n}\right)$. Then the automorphism $\sigma_{s}$ is as in (11) and $\sigma_{s} \in \operatorname{st}\left(L_{n}\right)$.

It is obvious that the map

$$
\mathbb{Z}_{p}^{n} \rightarrow \operatorname{st}\left(L_{n}\right), \quad s \mapsto \sigma_{s},
$$

is a group monomorphism. In fact, this map is an isomorphism; see Theorem 2.4, Before giving the proof of Theorem 2.4, we need to prove some more results.

Lemma 2.1. The kernel of the $\mathbb{F}_{p}$-linear map $\partial_{i}^{p-1}+F$ acting on the ring $L_{n}$ is $\mathbb{F}_{p} x_{i}^{-1}$, where $F: a \mapsto a^{p}$ is the Frobenius map on $L_{n}$.

Proof. First, let us consider the case when $i=n=1$. We drop the subscript 1 in this case. Let $s=\lambda x^{j}+\cdots \in K\left[x^{ \pm 1}\right]$ be a nonzero element of the kernel of the $\mathbb{F}_{p}$-linear map $\partial^{p-1}+F$, where $\lambda x^{j}$ is its least term, $0 \neq \lambda \in K$ (the map $\partial_{i}^{p-1}+F$ is $\mathbb{F}_{p}$-linear since $F(\mu)=\mu^{p}=\mu$ for all $\left.\mu \in \mathbb{F}_{p}\right)$. Then

$$
0=\left(\partial^{p-1}+F\right)\left(\lambda x^{j}\right)=\lambda j(j-1) \cdots(j-p+2) x^{j-p+1}+\lambda^{p} x^{p j},
$$

and so $j-p+1=p j$ and $\lambda j(j-1) \cdots(j-p+2)+\lambda^{p}=0$. The first equality yields $j=-1$. Then the second can be written as $\lambda^{p}-\lambda=0$ since $(p-1) ! \equiv-1$ $\bmod p$. Therefore, $\lambda \in \mathbb{F}_{p}$ and the set $\mathbb{F}_{p} x^{-1}$ belongs to the kernel of the map $\partial^{p-1}+F$. The least term of the element $s-\lambda x^{-1}$ of the kernel of the map $\partial^{p-1}+F$ 
has to be zero by the above argument, and so $s-\lambda x^{-1}=0$. This proves that $\operatorname{ker}\left(\partial^{p-1}+F\right)=\mathbb{F}_{p} x^{-1}$. Now, the general case follows from this special one since $L_{n} \subset K\left(x_{1}, \ldots, x_{i-1}, x_{i+1}, \ldots, x_{n}\right)\left[x_{i}^{ \pm 1}\right]$.

Let $R$ be a ring. The first Weyl algebra $A_{1}(R)$ over $R$ is a ring generated over $R$ by two elements $x$ and $\partial$ that satisfy the defining relation $\partial x-x \partial=1$.

Theorem 2.2 ([5]). Let $K$ be a reduced commutative $\mathbb{F}_{p}$-algebra and $A_{1}(K)$ be the first Weyl algebra over $K$. Then

$$
(\partial+f)^{p}=\partial^{p}+\frac{d^{p-1} f}{d x^{p-1}}+f^{p}
$$

for all $f \in K[x]$. In more detail, $(\partial+f)^{p}=\partial^{p}-\lambda_{p-1}+f^{p}$, where $f=\sum_{i=0}^{p-1} \lambda_{i} x^{i} \in$ $K[x]=\bigoplus_{i=0}^{p-1} K\left[x^{p}\right] x^{i}, \lambda_{i} \in K\left[x^{p}\right]$.

Corollary 2.3. For each element $f \in L_{n}$ and $i=1, \ldots, n$, the equality

$$
\left(\partial_{i}+f\right)^{p}=\frac{\partial^{p-1} f}{\partial x_{i}^{p-1}}+f^{p}
$$

holds in the ring $\mathcal{D}\left(L_{n}\right)$.

Proof. Let $L$ be the subalgebra $K\left[x_{1}^{ \pm 1}, \ldots, x_{i-1}^{ \pm 1}, x_{i}^{ \pm p}, x_{i+1}^{ \pm 1}, \ldots, x_{n}^{ \pm 1}\right]$ of the algebra $L_{n}$. Then $L_{n}=\bigoplus_{j=0}^{p-1} L x_{i}^{j}$. Consider the $L$-algebra homomorphism

$$
A_{1}(L):=L\langle x, \partial\rangle \rightarrow \mathcal{D}\left(L_{n}\right), \quad x \mapsto x_{i}, \quad \partial \mapsto \partial_{i}, \quad l \mapsto l,
$$

where $l \in L$. Now, the result follows from Theorem 2.2 since $\partial_{i}^{p}=0$.

$$
\Delta_{n}:=\bigoplus_{\alpha \in \mathbb{N}^{n}} K \partial^{[\alpha]} \text { is the algebra of scalar differential operators on } P_{n} \text {. }
$$

Theorem 2.4. The map (13) is an isomorphism.

Proof. It suffices to show that, for each automorphism $\sigma \in \operatorname{st}\left(L_{n}\right)$,

$$
\cdots \sigma_{p^{k} s_{k}} \cdots \sigma_{p s_{1}} \sigma_{s_{0}} \sigma=1
$$

for some elements $s_{k}=\left(s_{k 1}, \ldots, s_{k n}\right) \in\{0,1, \ldots, p-1\}^{n}$. Note that for all $k \geq 0$ and $i=1, \ldots, n$,

$$
\sigma_{p^{k} s_{k}}\left(\partial_{i}^{\left[p^{s}\right]}\right)=\partial_{i}^{\left[p^{s}\right]}, \quad s<k ; \quad \sigma_{p^{k} s_{k}}\left(\partial_{i}^{\left[p^{k}\right]}\right)=\partial_{i}^{\left[p^{s} k\right]}+s_{k i} x_{i}^{-p^{k}} .
$$

Note that the centralizer $C\left(\mathcal{D}\left(L_{n}\right), x_{1}, \ldots, x_{n}\right)$ of the elements $x_{1}, \ldots, x_{n}$ in the ring $\mathcal{D}\left(L_{n}\right)$ satisfies

$$
C\left(\mathcal{D}\left(L_{n}\right), x_{1}, \ldots, x_{n}\right):=\bigcap_{i=1}^{n} \operatorname{ker}\left(\operatorname{ad}\left(x_{i}\right)\right)=L_{n},
$$

where $\operatorname{ad}\left(x_{i}\right)$ is the inner derivation of the ring $\mathcal{D}\left(L_{n}\right)$ determined by the element $x_{i}$. For all indices $i$ and $j$,

$$
\left[\sigma\left(\partial_{i}\right)-\partial_{i}, x_{j}\right]=\sigma\left(\left[\partial_{i}, x_{j}\right]\right)-\left[\partial_{i}, x_{j}\right]=\delta_{i j}-\delta_{i j}=0 ;
$$

hence $a_{i}:=\sigma\left(\partial_{i}\right)-\partial_{i} \in L_{n}$. Using Corollary 2.3, we see that

$$
0=\sigma\left(\partial_{i}^{p}\right)=\left(\partial_{i}+a_{i}\right)^{p}=\left(\partial_{i}^{p-1}+F\right)\left(a_{i}\right),
$$

and so $a_{i}=-s_{0 i} x_{i}^{-1}$ for some elements $s_{0 i} \in \mathbb{F}_{p}$, by Lemma 2.1. Abusing the notation we assume in this proof that $\mathbb{F}_{p}=\{0,1, \ldots, p-1\}$. Let $s_{0}:=\left(s_{01}, \ldots s_{0 n}\right)$. 
Then $\sigma_{s_{0}} \sigma\left(\partial_{i}\right)=\partial_{i}$ for all $i$. Suppose that $k>0$, and we have found vectors $s_{0}, \ldots, s_{k-1} \in \mathbb{F}_{p}^{n}$ such that

$$
\sigma_{p^{k-1} s_{k-1}} \cdots \sigma_{p s_{1}} \sigma_{s_{0}} \sigma\left(\partial_{i}^{\left[p^{s}\right]}\right)=\partial_{i}^{\left[p^{s}\right]}, \quad 1 \leq i \leq n, \quad 0 \leq s \leq k-1 .
$$

In order to finish the proof of the claim by induction on $k$, we have to find a vector $s_{k} \in \mathbb{F}_{p}^{n}$ such that the above equalities hold for $k$ rather than $k-1$. Let $\tau:=\sigma_{p^{k-1} s_{k-1}} \cdots \sigma_{p s_{1}} \sigma_{s_{0}} \sigma$. For all $i$ and $j$,

$$
\left[\tau\left(\partial_{i}^{\left[p^{k}\right]}\right)-\partial_{i}^{\left[p^{k}\right]}, x_{j}^{p^{k}}\right]=\tau\left(\left[\partial_{i}^{\left[p^{k}\right]}, x_{j}^{p^{k}}\right]\right)-\left[\partial_{i}^{\left[p^{k}\right]}, x_{j}^{p^{k}}\right]=\delta_{i j}-\delta_{i j}=0 ;
$$

hence

$$
b_{i}:=\tau\left(\partial_{i}^{\left[p^{k}\right]}\right)-\partial_{i}^{\left[p^{k}\right]} \in C\left(\mathcal{D}\left(L_{n}\right), x_{1}^{p^{k}}, \ldots x_{n}^{p^{k}}\right)=L_{n} \otimes \Delta_{n,<p^{k}},
$$

where $\Delta_{n,<p^{k}}=\bigoplus_{\text {all } \alpha_{i}<p^{k}} K \partial^{[\alpha]}$. For all $i \neq j$,

$$
0=\tau\left(\left[\partial_{i}^{\left[p^{k}\right]}, x_{j}\right]\right)=\left[\partial_{i}^{\left[p^{k}\right]}+b_{i}, x_{j}\right]=\left[b_{i}, x_{j}\right],
$$

and so $b_{i} \in \sum_{s<p^{k}} L_{n} \partial_{i}^{[s]}$. Now,

$$
\partial_{i}^{\left[p^{k}-1\right]}=\tau\left(\partial_{i}^{\left[p^{k}-1\right]}\right)=\tau\left(\left[\partial_{i}^{\left[p^{k}\right]}, x_{i}\right]\right)=\left[\partial_{i}^{\left[p^{k}\right]}+b_{i}, x_{i}\right]=\partial_{i}^{\left[p^{k}-1\right]}+\left[b_{i}, x_{i}\right] ;
$$

hence $b_{i} \in L_{n}$. For all $i$ and $j$, and $s<k$,

$$
0=\tau\left(\left[\partial_{i}^{\left[p^{k}\right]}, \partial_{j}^{\left[p^{s}\right]}\right]\right)=\left[\partial_{i}^{\left[p^{k}\right]}+b_{i}, \partial_{j}^{\left[p^{s}\right]}\right]=\left[b_{i}, \partial_{j}^{\left[p^{s}\right]}\right],
$$

and so $b_{i} \in K\left[x_{1}^{ \pm p^{k}}, \ldots, x_{n}^{ \pm p^{k}}\right]$. Finally,

$$
0=\tau\left(\partial_{i}^{\left[p^{k}\right] p}\right)=\left(\partial_{i}^{\left[p^{k}\right]}+b_{i}\right)^{p}=\left(\partial_{i}^{\left[p^{k}\right](p-1)}+F\right)\left(b_{i}\right) ;
$$

hence $b_{i}=-s_{k i} x_{i}^{p^{k}}$ for some elements $s_{k i} \in \mathbb{F}_{p}$, by Lemma 2.1. Let $s_{k}=$ $\left(s_{k 1}, \ldots, s_{k n}\right)$. Then

$$
\sigma_{p^{k} s_{k}} \cdots \sigma_{p s_{1}} \sigma_{s_{0}} \sigma\left(\partial_{i}^{\left[p^{s}\right]}\right)=\partial_{i}^{\left[p^{s}\right]}, \quad 1 \leq i \leq n, \quad 0 \leq s \leq k .
$$

This finishes the proof of the theorem.

Corollary 2.5. Aut ord $\left(\mathcal{D}\left(L_{n}\right)\right)=\operatorname{St}\left(L_{n}\right)$.

Proof. The inclusion $\operatorname{Aut}_{\text {ord }}\left(\mathcal{D}\left(L_{n}\right)\right) \subseteq \operatorname{St}\left(L_{n}\right)$ is obvious; see (5). The inverse inclusion follows from the facts that $\operatorname{St}\left(L_{n}\right)=\operatorname{st}\left(L_{n}\right) \rtimes \operatorname{Aut}_{K}\left(L_{n}\right), \operatorname{st}\left(L_{n}\right) \subseteq$ $\operatorname{Aut}_{\text {ord }}\left(\mathcal{D}\left(L_{n}\right)\right)$ (by Theorem 2.4) and $\operatorname{Aut}_{K}\left(L_{n}\right) \subseteq \operatorname{Aut}_{\text {ord }}\left(\mathcal{D}\left(L_{n}\right)\right)$; see (3).

Proof of Theorem 1.3. This follows from Corollary 2.5, (4) and Theorem 2.4

$$
\operatorname{Aut}_{\text {ord }}\left(\mathcal{D}\left(L_{n}\right)\right)=\operatorname{St}\left(L_{n}\right)=\operatorname{st}\left(L_{n}\right) \rtimes \operatorname{Aut}_{K}\left(L_{n}\right) \simeq \mathbb{Z}_{p}^{n} \rtimes \operatorname{Aut}_{K}\left(L_{n}\right) .
$$

\section{REFERENCES}

[1] H. Bass, E. H. Connell and D. Wright, The Jacobian Conjecture: Reduction of degree and formal expansion of the inverse, Bull. Amer. Math. Soc. (New Series), 7 (1982), 287-330. MR663785 (83k:14028)

[2] V. V. Bavula, The inversion formulae for automorphisms of polynomial algebras and differential operators in prime characteristic, J. Pure Appl. Algebra, 212 (2008), 2320-2337. MR.2426512

[3] V. V. Bavula, Extensions of the Frobenius to ring of differential operators on polynomial algebra in prime characteristic, arXiv:math.RA/0804.1091. 
[4] V. V. Bavula, The Jacobian Conjecture ${ }_{2 n}$ implies the Dixmier Problem ${ }_{n}$, arXiv:math. RA/0512250

[5] V. V. Bavula, The group of automorphisms of the first Weyl algebra in prime characteristic and the restriction map, Glasgow Math. J., to appear (arXiv:math.RA/0708.1620).

[6] A. Belov-Kanel and M. Kontsevich, The Jacobian conjecture is stably equivalent to the Dixmier Conjecture, Mosc. Math. J., 7 (2007), no. 2, 209-218. MR.2337879

[7] J. Dixmier, Sur les algèbres de Weyl. Bull. Soc. Math. France, 96 (1968), 209-242. MR0242897 (39:4224)

[8] H. W. E. Jung, Uber ganze birationale Transformationen der Ebene, J. Reine Angew. Math., 184 (1942), 161-174. MR0008915 (5:74f)

[9] Y. Tsuchimoto, Endomorphisms of Weyl algebra and p-curvatures. Osaka J. Math., 42 (2005), no. 2, 435-452. MR 2147727 (2006g:14101)

[10] W. van der Kulk, On polynomial rings in two variables, Nieuw. Arch. Wisk. (3) 1 (1953), 33-41. MR.0054574 (14:941f)

Department of Pure Mathematics, University of Sheffield, Hicks Building, Sheffield S3 7RH, United Kingdom

E-mail address: v.bavula@sheffield.ac.uk 